SCHOOL OF OPERATIONS RESEARCH AND INDUSTRIAL ENGINEERING

COLLEGE OF ENGINEERING

CORNELL UNIVERSITY

ITHACA, NEW YORK 14853-3801

TECHNICAL REPORT NO. 1390

November 2003

\title{
OPTIMAL SELLING RULES IN A REGIME SWITCHING MODEL
}

by

X. Guo and Q. Zhang 


\title{
Optimal selling rules in a regime switching model
}

\author{
$\begin{array}{ll}\text { X. Guo } & \text { Q. Zhang } \\ & \end{array}$
}

\begin{abstract}
In this paper, we derive optimal selling rules for an investor holding one (share of) stock in a market which fluctuates among several (for example, "bull" and "bear") states. The mathematical model for stock fluctuations is a regime switching one: a set of the established Black-Scholes models coupled with a finite state continuous Markov chain. The optimal stopping rule is of a threshold type for each state, derived via the "modified smooth fit". The proof is via the martingale theory. Numerical examples are reported to demonstrate the dependence of threshold levels with various parameters and to compare our result with some sub-optimal selling rules.
\end{abstract}

Keywords: Markov process, Regime switching, Smooth fit, Optimal stopping

\section{Introduction}

Imagine an investor, who is holding one (share of) stock whose value $X(t)$ at time $t$ is random. Assume (not unrealistically) that the underlying market fluctuates between a bull market and a bear market. His goal is to decide a "best" selling time to maximize his discounted (due to inflations) expected payoff. Assume also that he is not clairvoyant.

This is a typical optimal stopping time problem, with the stopping time $\tau \leq t$ measurable to the "information" (the $\sigma$-algebra generated by $X(s)$ ) available up to time $t$. Clearly, the choice of an optimal stopping rule will be dictated by both the underlying model for $X(\cdot)$ and the payoff function.

A special case of this problem was studied by H. McKean [4] in the 1960's. He showed that if (a) one assumes that the fluctuation of $X(t)$ is driven by a geometric Brownian motion (now known as the Black-Scholes model) such that $d X(t)=\mu X(t) d t+\sigma X(t) d W(t)$, (b) the payoff function at time $t$ is $(X(t)-K)$ (or equivalently $(X(t)-K)^{+}$), with $K$ understood as an obligation to pay back when he sells the stock, and (c) there is a discounted factor $r$, so that one would like to maximize $E\left[e^{-r \tau}(X(\tau)-K)\right]$ over all possible choices of stopping times. Then if $\mu>r$, one can patiently

*School of Operations Research and Industrial Engineering, Cornell University, Ithaca, NY 14853

${ }^{\dagger}$ Department of Mathematics, University of Georgia, Athens, GA 30602 
"wait and see" and the return is infinite; if $\mu<r$, the optimal stopping strategy is of a threshold type. More precisely, there exists an $x^{*}$ which is explicitly given in terms of $\mu, r, \sigma, K$ so that the optimal stopping time $\tau^{*}=\inf \left\{t>0, X(t) \geq x^{*}\right\} ; \mu=r$ is a degenerate case for which the value function is $x$ with the corresponding $\tau^{*}=\infty, a . s$.

McKean's approach solves the special case for the question raised by our investor, namely when there is no state change in the market and parameters $\mu$ and $\sigma$ are constant. To answer the question for the general case with regime changes in the market, we shall consider the following switching diffusion process

$$
d X(t)=X(t) \mu_{\epsilon(t)} d t+X(t) \sigma_{\epsilon(t)} d W(t), X(0)=x
$$

where $\epsilon(t) \in\{1,2, \cdots, S\}$ is a finite-state continuous Markov chain, and $W(t)$ is a standard Wiener process defined on a probability space $(\Omega, \mathcal{F}, P)$. Here we assume that $W(\cdot)$ and $\epsilon(\cdot)$ are independent, and $\mu_{i}$ and $\sigma_{i}$ are known parameters for any given $\epsilon(t)=i$.

This model is in general referred to as a regime switching model and has been studied in various contexts; see for example, Di Masi, Kabanov and Runggaldier [3], Guo [2], and Zhang [8] among others. Two most relevant ones are [2] and [8]. The former dealt with a perpetual lookback option pricing and solved a related optimal stopping time problem via extending the technique of the well-known smooth fit; while the latter used a two-point-boundary-value approach and provided a suboptimal selling rule involving target and cut-loss levels, which is optimal in that particular context.

In this paper, we will exploit the technique developed in [2] for the optimal stopping problem assuming the payoff function $(X(t)-K)$ under the regime switching model (1). For explicitness, we assume that $S=2$ and the generator of $\epsilon(t)$ is of the form $\left(\begin{array}{cc}-\lambda_{1} & \lambda_{1} \\ \lambda_{2} & -\lambda_{2}\end{array}\right)$, with $\lambda_{1}, \lambda_{2}>0$. We will derive explicitly a general optimal selling rule and the corresponding value function in a closedform. We show that when $r<B_{1}\left(=\left(\mu_{1}-\lambda_{1}+\mu_{2}-\lambda_{2}+\sqrt{\left.\left[\mu_{1}-\lambda_{1}\right)-\left(\mu_{2}-\lambda_{2}\right)\right]^{2}+4 \lambda_{1} \lambda_{2}}\right) / 2\right)$, the best bet is to wait and get an infinite return; when $r>B_{1}$, the optimal stopping rule is of a threshold type for each state; $r=B_{1}$ is the degenerate case for which the value is $x$ and the waiting time is infinite. The proof of optimality is via the Dynkin's formula and local martingales. Finally, we illustrate numerically the dependence of our optimal threshold levels with various parameters and the difference between our solution and that in [8]. 


\section{Main results}

Mathematically, we are to consider the following optimal stopping problem in a state space $(x, \epsilon)$ :

$$
V_{i}^{*}(x)=\sup _{0 \leq \tau \leq \infty} E\left[e^{-r \tau}(X(\tau)-K) \mid X(0)=x, \epsilon(0)=i\right]
$$

where $\tau$ is an $\mathcal{F}_{t}=\sigma\{(W(s), \epsilon(s)) \mid s \leq t\}$-stopping time.

Intuitively, if $r$ is very small so that $e^{r t}$ is smaller than $E\left[X_{t}\right]$, it does not hurt to wait and the payoff is infinite. We will see that this is indeed true from the following lemma.

Lemma 1 When $\lambda_{1}, \lambda_{2}>0$,

$$
\begin{aligned}
& M_{1}(t)=E\left[\exp \int_{0}^{t} \mu_{\epsilon}(s) d s \mid \epsilon(0)=1\right]=\frac{\mu_{1}-B_{2}}{B_{1}-B_{2}} e^{B_{1} t}+\frac{B_{1}-\mu_{1}}{B_{1}-B_{2}} e^{B_{2} t}, \\
& M_{2}(t)=E\left[\exp \int_{0}^{t} \mu_{\epsilon}(s) d s \mid \epsilon(0)=2\right]=\frac{\mu_{2}-B_{2}}{B_{1}-B_{2}} e^{B_{1} t}+\frac{B_{1}-\mu_{2}}{B_{1}-B_{2}} e^{B_{2} t} .
\end{aligned}
$$

If $\lambda_{1}=0$, then $M_{1}(t)=\exp \left(\mu_{1} t\right)$, and

$$
M_{2}(t)=\frac{\mu_{1}-\mu_{2}}{\mu_{1}-\mu_{2}+\lambda_{2}} \exp \left(\left(\mu_{2}-\lambda_{2}\right) t\right)+\frac{\lambda_{2}}{\mu_{1}-\mu_{2}+\lambda_{2}} \exp \left(\mu_{1} t\right) .
$$

If $\lambda_{2}=0$, then $M_{2}(t)=\exp \left(\mu_{2} t\right)$, and

$$
M_{1}(t)=\frac{\mu_{2}-\mu_{1}}{\mu_{2}-\mu_{1}+\lambda_{1}} \exp \left(\left(\mu_{1}-\lambda_{1}\right) t\right)+\frac{\lambda_{1}}{\mu_{2}-\mu_{1}+\lambda_{1}} \exp \left(\mu_{2} t\right) .
$$

Here $B_{1}>0>B_{2}$ are the roots of the quadratic equation

$$
x^{2}-\left(\mu_{1}-\lambda_{1}+\mu_{2}-\lambda_{2}\right) x+\left(\mu_{1}-\lambda_{1}\right)\left(\mu_{2}-\lambda_{2}\right)-\lambda_{1} \lambda_{2}=0
$$

such that

$$
B_{1,2}=\frac{\mu_{1}-\lambda_{1}+\mu_{2}-\lambda_{2} \pm \sqrt{\left.\left[\mu_{1}-\lambda_{1}\right)-\left(\mu_{2}-\lambda_{2}\right)\right]^{2}+4 \lambda_{1} \lambda_{2}}}{2}
$$

Proof. Starting from time 0 and state 1 , between time 0 and $\Delta t$, there is either at least one jump from state 1 to 2 , with probability $1-e^{-\lambda_{1} \Delta t}$; or no jump and $\epsilon$ remains in state 1 with probability $e^{-\lambda_{1} \Delta t}$ and all the process starts afresh again. Since $\int_{0}^{t} d t=\int_{0}^{\Delta t} d t+\int_{\Delta t}^{t} d t$, it is clear that

$$
\begin{aligned}
M_{1}(t)= & E\left[\exp \int_{0}^{t} \mu_{\epsilon}(s) d s\left\{1_{(\text {jumps })}+1_{(\text {no jumps })}\right\}\right] \\
= & P\{\text { no jump }\} \exp \left(\mu_{1} \Delta t\right) M_{1}(t-\Delta t) \\
& +P\{\text { jumps }\} M_{2}(t+\Delta t * \eta)+O\left((\Delta t)^{2}\right) \\
= & \exp \left(-\lambda_{1} \Delta t\right) \exp \left(\mu_{1} \Delta t\right) M_{1}(t-\Delta t) \\
& +\left(1-e^{-\lambda_{1} \Delta t}\right) M_{2}(t+\Delta t * \eta)+O\left((\Delta t)^{2}\right)
\end{aligned}
$$


with $|\eta| \leq 1$. Similarly,

$$
\begin{aligned}
M_{2}(t)= & \exp \left(-\lambda_{2} \Delta t\right) \exp \left(\mu_{2} \Delta t\right) M_{2}(t-\Delta t) \\
& +\left(1-e^{-\lambda_{2} \Delta t}\right) M_{1}\left(t+\Delta t * \eta^{\prime}\right)+O\left((\Delta t)^{2}\right),
\end{aligned}
$$

with $\left|\eta^{\prime}\right| \leq 1$.

By a Taylor's series expansion and some algebra, we get the corresponding ODEs system with initial conditions for $M_{1}(t), M_{2}(t)$ such that

$$
\begin{aligned}
& \lambda_{1} M_{2}(t)=-\left(\mu_{1}-\lambda_{1}\right) M_{1}(t)+M_{1}^{\prime}(t), M_{1}(0)=1, \quad M_{1}^{\prime}(0)=\mu_{1} \\
& \lambda_{2} M_{1}(t)=-\left(\mu_{2}-\lambda_{2}\right) M_{2}(t)+M_{2}^{\prime}(t), M_{2}(0)=1, \quad M_{2}^{\prime}(0)=\mu_{2} .
\end{aligned}
$$

After simple calculations we see

$$
M_{1}^{\prime \prime}(t)-\left(\mu_{1}-\lambda_{1}+\mu_{2}-\lambda_{2}\right) M_{1}^{\prime}(t)+\left(\left(\mu_{1}-\lambda_{1}\right)\left(\mu_{2}-\lambda_{2}\right)-\lambda_{1} \lambda_{2}\right) M_{1}=0
$$

with the initial conditions $M_{1}(0)=1, M_{1}^{\prime}(0)=\mu_{1}$.

Solving this second order ODE with the form of $M_{1}(t)=\exp (B t)$ gives us

$$
M_{1}(t)=A_{1} \exp \left(B_{1} t\right)+A_{2} \exp \left(B_{2} t\right)
$$

Here $A_{i}^{\prime} s$ are uniquely determined by the boundary conditions such that $A_{1}=\left(\mu_{1}-B_{2}\right) /\left(B_{1}-B_{2}\right)$ and $A_{2}=\left(B_{1}-\mu_{1}\right) /\left(B_{1}-B_{2}\right)$.

$M_{2}(t)$ and the degenerate cases when $\lambda_{1} \lambda_{2}=0$ can be solved in a similar fashion.

\subsection{Case 1: $r \leq B_{1}$}

From the above lemma, together with the fact that $\exp \left(\int_{0}^{t} \sigma_{\epsilon} d B_{s}-\frac{1}{2} \int_{0}^{t} \sigma_{\epsilon}^{2} d s\right)$ is a martingale, it is immediate that $\left\{e^{-r t} X_{t}\right\}_{t \geq 0}$ is submartingale if $B_{1}>r$, and supermartingale if $B_{1}<r$. Moreover, we see

$$
\lim _{t \rightarrow \infty} E\left[e^{-r t} X_{t}\right]=\lim _{t \rightarrow \infty} e^{\left(B_{1}-r\right) t} X_{0}= \begin{cases}\infty & \left(B_{1}>r\right) \\ x & \left(B_{1}=r\right) \\ 0 & \left(B_{1}<r\right),\end{cases}
$$

and for $T<\infty$ and any stopping time $\tau$

$$
E\left[e^{-r(\tau \wedge T)} X_{\tau \wedge T}\right]-e^{-r(\tau \wedge T)} K=E\left[e^{-r \tau \wedge T}\left(X_{\tau \wedge T}-K\right)\right] \leq E\left[e^{-r(\tau \wedge T)} X_{\tau \wedge T}\right]
$$


Therefore, it is clear that if $B_{1}>r, \tau^{*}=\infty$ and the value function is infinite. When $B_{1}=r$, $\tau^{*}=\infty$ and the value function is $x$. This is easy from the optional sampling theorem.

Theorem 1 For $\left.\lambda_{1}>0, \lambda_{2}>0, V^{*}(x, i)\right)<\infty$ if and only if $r \geq B_{1}$. In particular, when $r=B_{1}$, $V^{*}(x, i)=x$

Remark 1 Note that the sufficient and necessary conditions for Theorem 1 only hold under the assumption $\lambda_{1} \lambda_{2} \neq 0$. When $\lambda_{1} \lambda_{2}=0$, it is possible that $\min \left(V_{1}(x), V_{2}(x)\right)<\infty$ and $\max \left(V_{1}(x), V_{2}(x)\right)=$ $\infty$, and vice versa. One can easily check one such example with $\lambda_{1}=0$, and $r \in\left(\mu_{1}, \mu_{2}-\lambda_{2}\right)$.

\subsection{Case 2: $r>B_{1}$}

In this case, the value function is finite. Therefore it is crucial to find the optimal stopping time $\tau$. If we focus our attention on the threshold type (the optimality of which is to be verified) because $(X(t), \epsilon)$ is jointly Markovian, then since $\epsilon$ changes from one state to another, it is reasonable to anticipate that the thresholds should be different according to the state of $\epsilon$.

Denoting each threshold $x_{i}$ for the state $i$, we will first provide our intuitive derivation of such $x_{i}$, and then to prove via the martingale theory the optimality of these $x_{i}$.

First, consider the case when $x_{1} \neq x_{2}$, and without loss of generality assume $x_{1}<x_{2}$. By the very definition of $x_{i}$, we know if $x>x_{1}$, one should stop if it is in state 1 , therefore $V_{2}(x)=x-K$; however, if it is in state 2 and $x<x_{2}$, we will hold the stock and watch the process $X(t)$ to continue. Note that over the next time interval $\delta t$, with probability $\lambda_{1} \delta t$, the state may change to state 2 for which the value function will become $V_{2}(x+\delta x)$, and with probability $1-\lambda_{1} \delta t$ the state may stay state 1 for which the value function will become $V_{1}(x+\delta x)$. If our strategy is "optimal", it is not hard to convince ourselves that we should have for small $\delta t$,

$$
V_{1}(x)=e^{-r \delta t}\left\{\lambda_{1} d t V_{2}(x+\delta x)+\left(1-\lambda_{1} d t\right) V_{1}(x+\delta x)\right\}
$$

Now assuming the value functions are smooth enough, then the Ito's calculus yields, for $x \in\left[x_{1}, x_{2}\right]$,

$$
\begin{cases}\left(r+\lambda_{2}\right) V_{2}(x) & =x \mu_{2} V_{2}^{\prime}(x)+\frac{1}{2} x^{2} \sigma_{2}^{2} V_{2}^{\prime \prime}(x)+\lambda_{2}(x-K), \\ V_{1}(x) & =x-K .\end{cases}
$$

Similarly, for $x \in\left[0, x_{1}\right)$,

$$
\left\{\begin{array}{l}
\left(r+\lambda_{1}\right) V_{1}(x)=x \mu_{1} V_{1}^{\prime}(x)+\frac{1}{2} x^{2} \sigma_{1}^{2} V_{1}^{\prime \prime}(x)+\lambda_{1} V_{2}(x) \\
\left(r+\lambda_{2}\right) V_{2}(x)=x \mu_{2} V_{2}^{\prime}(x)+\frac{1}{2} x^{2} \sigma_{2}^{2} V_{2}^{\prime \prime}(x)+\lambda_{2} V_{1}(x)
\end{array}\right.
$$


Finally, for $x \in\left[x_{2}, \infty\right]$, we have

$$
V_{1}(x)=V_{2}(x)=x-K
$$

Now assuming that $V_{1} \neq V_{2}$, clearly the characteristic equation associated with Eq. (8) is

$$
g_{1}(\beta) g_{2}(\beta)=\lambda_{1} \lambda_{2}
$$

where $g_{i}(\beta)=\lambda_{i}+r-\left(\mu_{i}-\frac{1}{2} \sigma_{i}^{2}\right) \beta-\frac{1}{2} \sigma_{i}^{2} \beta^{2},(i=1,2)$. This characteristics equation has four distinct roots $\beta_{1}<\beta_{2}<0<\beta_{3}<\beta_{4}$.

Therefore, the general form of the solution to Eq. (8) is given by

$$
\begin{aligned}
& V_{1}(x)=\sum_{i=1}^{4} A_{i} x^{\beta_{i}} \\
& V_{2}(x)=\sum_{i=1}^{4} l_{i} A_{i} x^{\beta_{i}}
\end{aligned}
$$

with $l_{i}=l\left(\beta_{i}\right)=\frac{g_{1}\left(\beta_{i}\right)}{\lambda_{1}}=\frac{\lambda_{2}}{g_{2}\left(\beta_{i}\right)}$, for $i=1,2,3,4$. Note that when $x \rightarrow 0, V_{i}(x)(i=1,2)$ are bounded. (More precisely, $V_{1}(0)=V_{2}(0)=0$.) Thus, the negative power of $x$ should be eliminated so that

$$
\begin{aligned}
& V_{1}(x)=A_{1} x^{\beta_{1}}+A_{2} x^{\beta_{2}}, \\
& V_{2}(x)=l_{1} A_{1} x^{\beta_{1}}+l_{2} A_{2} x^{\beta_{2}} .
\end{aligned}
$$

Next, we turn our attention to Eq. (7). We denote $\phi(x)$ as a special solution to

$$
\left(r+\lambda_{2}\right) V(x)=x \mu_{2} V^{\prime}(x)+\frac{1}{2} x^{2} \sigma_{2}^{2} V^{\prime \prime}(x)+\lambda_{2}(x-K)
$$

In particular, if $r+\lambda_{2}-\mu_{2} \neq 0$, one could take

$$
\phi(x)=-\frac{\lambda_{2} K}{r+\lambda_{2}}+\frac{\lambda_{2} x}{r+\lambda_{2}-\mu_{2}} .
$$

(In the case of $x_{1}>x_{2}$, we use $\widetilde{\phi}(x)$ as a special solution to

$$
\left(r+\lambda_{1}\right) V(x)=x \mu_{1} V^{\prime}(x)+\frac{1}{2} x^{2} \sigma_{1}^{2} V^{\prime \prime}(x)+\lambda_{1}(x-K)
$$

and choose when $r+\lambda_{1}-\mu_{1} \neq 0, \widetilde{\phi}(x)=-\frac{\lambda_{1} K}{r+\lambda_{1}}+\frac{\lambda_{1} x}{r+\lambda_{1}-\mu_{1}}$.)

Therefore, one can express, via $\phi(x)$ and $\gamma_{i}$, the general solution to Eq. (7) in the form of

$$
V_{2}(x)=C_{1} x^{\gamma_{1}}+C_{2} x^{\gamma_{2}}+\phi(x)
$$

with $\gamma_{i}(i=1,2)$ being the real roots of

$$
\mu_{2} \gamma+\frac{1}{2} \sigma_{2}^{2} \gamma(\gamma-1)=r+\lambda_{2}
$$


(For the case when $x_{1}>x_{2}$, we denote $\tilde{\gamma}_{i}(i=1,2)$ as the corresponding real roots of $\mu_{1} \gamma+$ $\left.\frac{1}{2} \sigma_{1}^{2} \gamma(\gamma-1)=r+\lambda_{1}.\right)$

In order to uniquely determine $V_{1}(x)$ and $V_{2}(x)$, we must solve for $A_{1}, A_{2}, C_{1}, C_{2}, x_{1}$, and $x_{2}$. To this end, we need appropriate boundary conditions.

Applying the smooth fit along the boundaries (i.e., at $x=x_{1}$ and $x=x_{2}$ ) with $V_{1}(x+)=V_{1}(x-$ ) and $V_{1}^{\prime}(x+)=V_{1}^{\prime}(x-)$ yields

$$
\left\{\begin{array}{l}
A_{1} x_{1}^{\beta_{1}}+A_{2} x_{1}^{\beta_{2}}=x_{1}-K \\
\beta_{1} A_{1} x_{1}^{\beta_{1}}+\beta_{2} A_{2} x_{1}^{\beta_{2}}=x_{1}
\end{array}\right.
$$

and the smoothness of $V_{2}(x)$ at $x_{1}$ and $x_{2}$ (the "modified smooth fit" [2]) suggests

$$
\left\{\begin{array}{l}
l_{1} A_{1} x_{1}^{\beta_{1}}+l_{2} A_{2} x_{1}^{\beta_{2}}=C_{1} x_{1}^{\gamma_{1}}+C_{2} x_{1}^{\gamma_{2}}+\phi\left(x_{1}\right) \\
l_{1} \beta_{1} A_{1} x_{1}^{\beta_{1}}+l_{2} \beta_{2} A_{2} x_{1}^{\beta_{2}}=\gamma_{1} C_{1} x_{1}^{\gamma_{1}}+\gamma_{2} C_{2} x_{1}^{\gamma_{2}}+x_{1} \phi^{\prime}\left(x_{1}\right)
\end{array}\right.
$$

and

$$
\begin{cases}C_{1} x_{2}^{\gamma_{1}}+C_{2} x_{2}^{\gamma_{2}}+\phi\left(x_{2}\right) & =x_{2}-K \\ \gamma_{1} C_{1} x_{2}^{\gamma_{1}}+\gamma_{2} C_{2} x_{2}^{\gamma_{2}}-x_{2} \phi^{\prime}\left(x_{2}\right) & =x_{2}\end{cases}
$$

Combining the above three equations together with some algebraic manipulation, we obtain

$$
\left(\begin{array}{cc}
x_{1}^{-\gamma_{1}} & 0 \\
0 & x_{1}^{-\gamma_{2}}
\end{array}\right) F_{1}\left(x_{1}, \phi\left(x_{1}\right)\right)=\left(\begin{array}{cc}
x_{2}^{-\gamma_{1}} & 0 \\
0 & x_{2}^{-\gamma_{2}}
\end{array}\right) F_{2}\left(x_{2}, \phi\left(x_{2}\right)\right)
$$

where

$$
F_{1}(x, g(x))=\left(\begin{array}{cc}
1 & 1 \\
\gamma_{1} & \gamma_{2}
\end{array}\right)^{-1}\left[\left(\begin{array}{cc}
l_{1} & l_{2} \\
l_{1} \beta_{1} & l_{2} \beta_{2}
\end{array}\right)\left(\begin{array}{cc}
1 & 1 \\
\beta_{1} & \beta_{2}
\end{array}\right)^{-1}\left(\begin{array}{c}
x-K \\
x
\end{array}\right)-\left(\begin{array}{c}
g(x) \\
x g^{\prime}(x)
\end{array}\right)\right]
$$

and

$$
\widetilde{F}_{1}(x, g(x))=\left(\begin{array}{cc}
1 & 1 \\
\gamma_{1} & \gamma_{2}
\end{array}\right)^{-1}\left[\left(\begin{array}{cc}
\tilde{l}_{1} & \widetilde{l}_{2} \\
\widetilde{l}_{1} \beta_{1} & \widetilde{l}_{2} \beta_{2}
\end{array}\right)\left(\begin{array}{cc}
1 & 1 \\
\beta_{1} & \beta_{2}
\end{array}\right)^{-1}\left(\begin{array}{c}
x-K \\
x
\end{array}\right)-\left(\begin{array}{c}
g(x) \\
x g^{\prime}(x)
\end{array}\right)\right]
$$

where $\tilde{l}_{i}=1 / l_{i}$ by symmetry. (Again, the corresponding notation when $x_{1}>x_{2}$ is given by

$$
F_{2}(x, g(x))=\left(\begin{array}{cc}
1 & 1 \\
\gamma_{1} & \gamma_{2}
\end{array}\right)^{-1}\left(\begin{array}{c}
x-K-g(x) \\
x-x g^{\prime}(x)
\end{array}\right)
$$

and

$$
\widetilde{F}_{2}(x, g(x))=\left(\begin{array}{cc}
1 & 1 \\
\widetilde{\gamma}_{1} & \widetilde{\gamma}_{2}
\end{array}\right)^{-1}\left(\begin{array}{c}
x-K-g(x) \\
x-x g^{\prime}(x)
\end{array}\right) .
$$

For the case $x_{1}=x_{2}=x^{*}$, it is not difficult to show that the value function reduces to the McKean's problem. Indeed, in this case,

$$
\begin{aligned}
& V_{1}(x)=A_{1} x^{\beta_{1}}+A_{2} x^{\beta_{2}} \\
& V_{2}(x)=l_{1} A_{1} x^{\beta_{1}}+l_{2} A_{2} x^{\beta_{2}}
\end{aligned}
$$


for $x \in\left[0, x^{*}\right]$ and $V_{1}(x)=V_{2}(x)=x-K$ for $x \geq x^{*}$. The smooth fit scheme leads to

$$
\left\{\begin{array}{l}
A_{1}\left(x^{*}\right)^{\beta_{1}}+A_{2}\left(x^{*}\right)^{\beta_{2}}=x^{*}-K \\
\beta_{1} A_{1}\left(x^{*}\right)^{\beta_{1}}+\beta_{2} A_{2}\left(x^{*}\right)^{\beta_{2}}=x^{*}
\end{array}\right.
$$

and

$$
\left\{\begin{array}{l}
l_{1} A_{1}\left(x^{*}\right)^{\beta_{1}}+l_{2} A_{2}\left(x^{*}\right)^{\beta_{2}}=x^{*}-K \\
l_{1} \beta_{1} A_{1}\left(x^{*}\right)^{\beta_{1}}+l_{2} \beta_{2} A_{2}\left(x^{*}\right)^{\beta_{2}}=x^{*}
\end{array}\right.
$$

Necessarily, we have $A_{1}=l_{1} A_{1}$ and $A_{2}=l_{2} A_{2}$. Which means $V_{1}=V_{2}$. This implies $\mu_{1}=\mu_{2}, \sigma_{1}=$ $\sigma_{2}$ from equation (8). And the value function can be easily rederived via the smooth fit, which is exactly the solution by McKean's approach.

In conclusion, if we use $Y_{1}, Y_{2}$ to denote column vectors and define

$$
H\left(x_{1}, x_{2}, Y_{1}, Y_{2}\right)=\left(\begin{array}{cc}
x_{1}^{-\gamma_{1}} & 0 \\
0 & x_{1}^{-\gamma_{2}}
\end{array}\right) Y_{1}-\left(\begin{array}{cc}
x_{2}^{-\gamma_{1}} & 0 \\
0 & x_{2}^{-\gamma_{2}}
\end{array}\right) Y_{2}
$$

and

$$
\widetilde{H}\left(x_{1}, x_{2}, Y_{1}, Y_{2}\right)=\left(\begin{array}{cc}
x_{1}^{-\tilde{\gamma}_{1}} & 0 \\
0 & x_{1}^{-\tilde{\gamma}_{2}}
\end{array}\right) Y_{1}-\left(\begin{array}{cc}
x_{2}^{-\widetilde{\gamma}_{1}} & 0 \\
0 & x_{2}^{-\widetilde{\gamma}_{2}}
\end{array}\right) Y_{2}
$$

Then we have

Theorem 2 Assuming $V_{i}^{*}(x) \in C^{1}(0, \infty)$, and $r>B_{1}$, then the optimal value functions $V_{i}^{*}(x)=$ $V_{i}(x)$ where $V_{i}(x)>x-K$ is determined by three cases:

Case 1: If there exists an $x_{1}<x_{2}$ such that $H\left(x_{1}, x_{2}, F_{1}\left(x_{1}, \phi\left(x_{1}\right)\right), F_{2}\left(x_{2}, \phi\left(x_{2}\right)\right)\right)=0$, then

$$
\begin{aligned}
& V_{1}(x)= \begin{cases}A_{1} x^{\beta_{1}}+A_{2} x^{\beta_{2}} & \text { if } x<x_{1}, \\
x-K & \text { if } x \geq x_{1},\end{cases} \\
& V_{2}(x)= \begin{cases}l_{1} A_{1} x^{\beta_{1}}+l_{2} A_{2} x^{\beta_{2}} & \text { if } x<x_{1}, \\
C_{1} x^{\gamma_{1}}+C_{2} x^{\gamma_{2}}+\phi(x) & \text { if } x_{2}>x \geq x_{1}, \\
x-K & \text { if } x \geq x_{2} .\end{cases}
\end{aligned}
$$

with

$$
\begin{gathered}
\left(\begin{array}{c}
A_{1} \\
A_{2}
\end{array}\right)=\left(\begin{array}{cc}
x_{1}^{\beta_{1}} & x_{1}^{\beta_{2}} \\
\beta_{1} x_{1}^{\beta_{1}} & \beta_{2} x_{1}^{\beta_{2}}
\end{array}\right)^{-1}\left(\begin{array}{c}
x_{1}-K \\
x_{1}
\end{array}\right), \\
\left(\begin{array}{c}
C_{1} \\
C_{2}
\end{array}\right)=\left(\begin{array}{cc}
x_{2}^{\gamma_{1}} & x_{2}^{\gamma_{2}} \\
\gamma_{1} x_{2}^{\gamma_{1}} & \gamma_{2} x_{2}^{\gamma_{2}}
\end{array}\right)^{-1}\left(\begin{array}{c}
x_{2}-K-\phi\left(x_{2}\right) \\
x_{2}-x_{2} \phi^{\prime}\left(x_{2}\right)
\end{array}\right) .
\end{gathered}
$$

Case 2: If there exists an $x_{1}>x_{2}$ such that $\widetilde{H}\left(x_{1}, x_{2}, \widetilde{F}_{2}\left(x_{1}, \widetilde{\phi}\left(x_{1}\right)\right), \widetilde{F}_{1}\left(x_{2}, \widetilde{\phi}\left(x_{2}\right)\right)\right)=0$, then

$$
\begin{gathered}
V_{1}(x)= \begin{cases}\widetilde{A}_{1} x^{\beta_{1}}+\widetilde{A}_{2} x^{\beta_{2}} & \text { if } x<x_{2}, \\
\widetilde{C}_{1} x^{\widetilde{\gamma}_{1}}+\widetilde{C}_{2} x^{\widetilde{\gamma}_{2}}+\widetilde{\phi}(x) & \text { if } x_{1}>x \geq x_{2}, \\
x-K & \text { if } x \geq x_{1},\end{cases} \\
V_{2}(x)= \begin{cases}l_{1} \widetilde{A}_{1} x^{\beta_{1}}+l_{2} \widetilde{A}_{2} x^{\beta_{2}} & \text { if } x<x_{2}, \\
x-K & \text { if } x \geq x_{2},\end{cases}
\end{gathered}
$$


with

$$
\left(\begin{array}{c}
\widetilde{A}_{1} \\
\widetilde{A}_{2}
\end{array}\right)=\left(\begin{array}{cc}
l_{1} x_{2}^{\beta_{1}} & l_{2} x_{2}^{\beta_{2}} \\
l_{1} \beta_{1} x_{2}^{\beta_{1}} & l_{2} \beta_{2} x_{2}^{\beta_{2}}
\end{array}\right)^{-1}\left(\begin{array}{c}
x_{2}-K \\
x_{2}
\end{array}\right),
$$

and

$$
\left(\begin{array}{c}
\widetilde{C}_{1} \\
\widetilde{C}_{2}
\end{array}\right)=\left(\begin{array}{cc}
x_{1}^{\widetilde{\gamma}_{1}} & x_{1}^{\widetilde{\gamma}_{2}} \\
\widetilde{\gamma}_{1} x_{1}^{\widetilde{\gamma}_{1}} & \widetilde{\gamma}_{2} x_{1}^{\widetilde{\gamma}_{2}}
\end{array}\right)^{-1}\left(\begin{array}{c}
x_{1}-K-\widetilde{\phi}\left(x_{1}\right) \\
x_{1}-x_{1} \widetilde{\phi}^{\prime}\left(x_{1}\right)
\end{array}\right) .
$$

Case 3, $x_{1}=x_{2}=x^{*}$ if and only if $\mu_{1}=\mu_{2}, \sigma_{1}=\sigma_{2}$, for which

$$
V_{1}(x)=V_{2}(x)= \begin{cases}\frac{\left(x^{*}\right)^{1-\beta}}{\beta} x^{\beta} & \text { if } x<x^{*} \\ x-K & \text { if } x \geq x^{*}\end{cases}
$$

where $x^{*}=\beta K /(\beta-1)$ ); and $\beta>0$ satisfies $r-\left(\mu_{1}-\frac{1}{2} \sigma_{1}^{2}\right) \beta-\frac{1}{2} \sigma_{1}^{2} \beta^{2}=0$ (or equivalently $\left.r-\left(\mu_{2}-\frac{1}{2} \sigma_{2}^{2}\right) \beta-\frac{1}{2} \sigma_{2}^{2} \beta^{2}=0\right)$.

Theorem 3 When $r>B_{1}$, the optimal stopping rule is dictated by

$$
\tau^{*}=\inf \left\{t>0 \mid(X(t), \epsilon(t))=\left(x_{i}, i\right)\right\}
$$

such that

$$
V_{i}^{*}(x)=V_{i}(x)=E\left[e^{-r \tau^{*}}\left(X\left(\tau^{*}\right)-K\right) \mid X(0)=x, \epsilon(0)=i\right] .
$$

\subsection{Proof of Theorems 2 and 3}

It is easy to see that $V_{i}(\infty)=0, i=1,2$, and

$$
D=\left\{(x, 1) \mid x \in\left(x_{1}, \infty\right)\right\} \cup\left\{(x, 2) \mid x \in\left(x_{2}, \infty\right)\right\}
$$

For any $v(x, i) \in C^{2}$, define

$$
\mathcal{L} v(x, i)=x \mu_{i} \frac{\partial v(x, i)}{\partial x}+\frac{1}{2} x^{2} \sigma_{i}^{2} \frac{\partial^{2} v(x, i)}{\partial x^{2}}+\lambda_{i}(v(x, 3-i)-v(x, i))-r v(x, i) .
$$

Let $v(x, i)=V_{i}(x)$. Then $\mathcal{L} v \leq 0$ except at $(x, i)=\left(x_{i}, i\right)$. From the uniform integrability of $e^{-r t} V(X(t), \epsilon(t))$ (directly from the proof of Proposition 3.2 in [2]) and the Dynkin's formula, we have

$$
E\left[d\left(e^{-r t} v(X(t), \epsilon(t))\right)\right]=E\left[e^{-r t} \mathcal{L} v(X(t), \epsilon(t)) d t\right]
$$

For any stopping time $\tau$, it follows,

$$
v(x, i) \geq E\left[e^{-r \tau} v(X(\tau), \epsilon(\tau))\right] \geq E\left[e^{-r \tau}(X(\tau)-K)\right]
$$


To show the optimality of $\tau^{*}$, note that if $\tau^{*}<\infty$, then $v\left(X\left(\tau^{*}\right), \epsilon\left(\tau^{*}\right)\right)=K-X\left(\tau^{*}\right)$. In this case, the Dynkin's formula yields $v(x, i)=E\left[e^{-r \tau^{*}}\left(K-X\left(\tau^{*}\right)\right)\right]$. Otherwise, let

$$
D_{k}=D \cap\{x<k\}, \text { for } k=1,2, \ldots
$$

Let $\tau_{k}=\inf \left\{t \geq 0 \mid(X(t), \epsilon(t)) \notin D_{k}\right\}$. Then it is easy to see $\tau_{k} \rightarrow \tau^{*}$ a.s. Moreover, as in Zhang $\left[8\right.$, Theorems 4.5 and 4.6], we see that, for each $k, \tau_{k}<\infty$ a.s. Using the definition of $\tau_{k}$, we have, for $k>K$,

$$
v\left(X\left(\tau_{k}\right), \epsilon\left(\tau_{k}\right)\right)=v\left(X\left(\tau_{k}\right), \epsilon\left(\tau_{k}\right)\right) I_{\left\{X\left(\tau_{k}\right)=k\right\}}+v\left(X\left(\tau_{k}\right), \epsilon\left(\tau_{k}\right)\right) I_{\left\{X\left(\tau_{k}\right)<k\right\}}
$$

Note that

$$
v\left(X\left(\tau_{k}\right), \epsilon\left(\tau_{k}\right)\right) I_{\left\{X\left(\tau_{k}\right)<k\right\}}=\left(K-X\left(\tau_{k}\right)\right) I_{\left\{X\left(\tau_{k}\right)<k\right\}} \leq K-X\left(\tau_{k}\right) .
$$

Moreover, note that $0 \leq v(x, i) \leq K$ and $e^{-r \tau_{k}} I_{\left\{X\left(\tau_{k}\right)=k\right\}} \rightarrow 0$, as $k \rightarrow \infty$, a.s. It follows that

$$
E\left[e^{-r \tau_{k}} v\left(X\left(\tau_{k}\right), \epsilon\left(\tau_{k}\right)\right) I_{\left\{X\left(\tau_{k}\right)=k\right\}}\right] \rightarrow 0
$$

Therefore, we have

$$
v(x, i) \leq E\left[e^{-r \tau_{k}} v\left(X\left(\tau_{k}\right), \epsilon\left(\tau_{k}\right)\right)\right] \rightarrow E\left[e^{-r \tau^{*}}\left(K-X\left(\tau^{*}\right)\right)\right]
$$

as $k \rightarrow \infty$. Combining with (30), we have

$$
v(x, i)=E\left[e^{-r \tau^{*}}\left(K-X\left(\tau^{*}\right)\right)\right]
$$

This completes the proof.

\section{Numerical simulation}

As was mentioned earlier, in Zhang [8] a two-point boundary value differential equation (TPBVDE) approach was used to derive an "optimal" selling rule for the threshold type stopping rules. This is however sub-optimal for our problem, because the feasible solution was chosen from a constrained and smaller set of stopping rules. In this section we report numerical experiments for comparing our analytical solutions with the TPBVDE solutions.

First, we take $r=3, \mu_{1}=2, \mu_{2}=1, K=1, \lambda_{1}=\lambda_{2}=5, \sigma_{1}=4, \sigma_{2}=2$, and examine our closed-form solution and those by the TPBVDE method. In this case, the threshold levels are given 



Figure 1: Value functions and comparisons

by $\left(x_{1}, x_{2}\right)=(9.97,3.88)$. In Figure 1 , we use $V^{\mathrm{e}}(x, i)$ and $V^{2 \mathrm{ptbd}}(x, i)$ to denote value functions for our optimal stopping and the two-point boundary value problems, respectively. The differences $V^{\mathrm{e}}(x, i)$ and $(x-K)$ in Figure 1 validate the basic structure of the optimal stopping in terms of threshold levels $\left(x_{1}, x_{2}\right)$.

Next, we examine the monotonicity of these threshold levels with respect to $\sigma_{1}, \lambda_{1}$, and $K$.

First, we vary $\sigma_{1}$ and keep all other parameters fixed. The resulting $\left(x_{1}, x_{2}\right)$ are listed in Table 1. Both threshold levels $x_{1}, x_{2}$ increase with the increase in $\sigma_{1}$. This shows that a larger $\sigma_{1}$ leads to a higher expected reward, and therefore a higher threshold levels.

\begin{tabular}{|c|c|c|c|c|c|}
\hline$\sigma_{1}$ & 2 & 3 & 4 & 5 & 6 \\
\hline$\left(x_{1}, x_{2}\right)$ & $(5.02,3.31)$ & $(7.10,3.60)$ & $(9.97,3.88)$ & $(13.74,4.15)$ & $(18.42,4.38)$ \\
\hline
\end{tabular}

Table 1. Dependency on $\sigma_{1}$

We then vary $\lambda_{1}$. The result in Table 2 implies that both $x_{1}$ and $x_{2}$ increase if $\lambda_{1}$ increases: this is because a larger $\lambda_{1}$ implies a shorter period for $\epsilon(t)$ staying at $\epsilon(t)=1$ and a smaller weight on $\sigma_{1}=4\left(>\sigma_{2}=2\right)$, which leads to smaller average volatility. 


\begin{tabular}{|c|c|c|c|c|c|}
\hline$\lambda_{1}$ & 3 & 4 & 5 & 6 & 7 \\
\hline$\left(x_{1}, x_{2}\right)$ & $(10.56,3.98)$ & $(10.25,3.92)$ & $(9.97,3.88)$ & $(9.72,3.83)$ & $(9.50,3.81)$ \\
\hline
\end{tabular}

Table 2. Dependency on $\lambda_{1}$

Finally we vary $K$. It suggests in Table 3 that both $x_{1}$ and $x_{2}$ increase in $K$ due to the fact that larger $K$ implies higher transaction cost and that needs to be compensated by higher samplewise return level.

\begin{tabular}{|c|c|c|c|c|c|}
\hline$K$ & 1 & 2 & 3 & 4 & 5 \\
\hline$\left(x_{1}, x_{2}\right)$ & $(9.98,3.88)$ & $(19.95,7.76)$ & $(29.97,11.62)$ & $(39.95,15.50)$ & $(49.94,19.37)$ \\
\hline
\end{tabular}

Table 3. Dependency on $K$

\section{References}

[1] X. Guo (2001): Inside Information and option pricings, Quantitative Finance (2001), 1: 38-44.

[2] X. Guo (2001): An explicit solution to an optimal stopping problem with regime switching, Journal of Applied Probability 38(2): 464-481.

[3] G. B. Di Masi, Yu, M. Kabanov and W. J. Runggaldier (1994): Mean-variance hedging of options on stocks with Markov volatility, Theory Probability and Its Applications 39(1): 173181.

[4] H. P. McKean (1965): A Free Boundary Problem for the Heat Equation Arising from a Problem in Mathematical Economics, Industrial Management Review 60: 32-39.

[5] D. Revuz and M. Yor (1991): Continuous Martingale and Brownian Motion, Springer-Verlag.

[6] H. Robbins, D. Sigmund and Y. Chow (1971): Great Expectations: The Theory of Optimal Stopping, Houghton-Nifflin.

[7] L. Shepp and A. Shiryayev (1993): Russian options: reduced regret, Annals of Applied Probability 3: 631-640.

[8] Q. Zhang (2001): Stock trading: an optimal selling rule, SIAM journal on Control and Optimization, 40: 67-84. 